\title{
Synergistic Antioxidant Activities of Eight Traditional Chinese Herb Pairs
}

\author{
Wen-Jian YAng, ${ }^{a}$ Da-Peng Li, ${ }^{*}, a, b$ Jin-Kui Li, ${ }^{a}$ Ming-Hua Li, ${ }^{a}{ }^{\text {Yi-Lun Chen }},{ }^{a}$ and Pei-Zheng Zhang ${ }^{a}$ \\ ${ }^{a}$ College of Food Sciences, Shandong Agricultural University; and ${ }^{b}$ State Key Laboratory of Crop Biology, College of Life \\ Sciences, Shandong Agricultural University; Tai'an, Shandong 271018, P. R. China. \\ Received June 23, 2008; accepted March 23, 2009
}

\begin{abstract}
Many Chinese therapeutic herbs that are traditionally used in combination demonstrate significantly better pharmacological effects when used in the combination than when used alone. However, the pharmacological mechanism for this synergism is still not well understood. In the present study, the antioxidant activities of six herbs ((Paeonia lactiflora (PL), Atractylodes macrocephala (AMA), Angelica sinensis (AS), Astragalus membranaceus (AME), Glycyrrhiza uralensis (GU) and Rheum officinale (RO)), which were historically combined into eight traditional Chinese herb pairs (TCHPs) (AME-AS, AME-AMA, AME-RO, AME-GU, AME-PL, PL-AS, PL-AMA and PL-GU), were investigated in vitro by assessing the 1,1-diphenyl-2-picryl hydrazine (DPPH)-radical scavenging abilities of the herbs. The results of this study showed that all eight TCHPs had a significantly larger scavenging capacity than would be expected from the theoretical sum of those of the respective constituent herbs $(\boldsymbol{p}<0.05)$. Furthermore, the AME-GU, AME-PL and AME-AMA pairs not only showed a significant synergistic effect in the DPPH scavenging assay, but they also demonstrated similar results in hydroxyl radical and superoxide radical anion scavenging assays. Interestingly, the AME-AMA combination had a significantly higher superoxide anion $(0.2 \mathrm{~g} / \mathrm{ml})$ and hydroxyl radical scavenging ability than the AME or AMA. The changes in the total phenolic and flavonoid contents were also investigated. Our study showed a significant correlation between the rate of enhancement in antioxidant capacity and the rate of increase in flavonoid content. Thus, the flavonoids are likely responsible for the synergistic effects present in TCHPs.
\end{abstract}

Key words herb pair; antioxidant capability; synergistic effect; phenolic; flavonoid

Oxidative stress is mainly induced by reactive oxygen species (ROS) such as hydroxyl radicals $(\cdot \mathrm{OH})$, hydrogen peroxide $\left(\mathrm{H}_{2} \mathrm{O}_{2}\right)$, and superoxide radical anions $\left(\mathrm{O}_{2}^{-}\right)$. The adverse effects of oxidative stress have been demonstrated in several groups of diseases, including cardiovascular disease and heart disease, ${ }^{1,2)}$ liver injury, ${ }^{3)}$ acute and chronic renal damage, ${ }^{4)}$ and cancer. ${ }^{5}$ These negative effects are mainly caused by the formation of free radicals and mediated by free radical damage to lipids, proteins, and DNA. ${ }^{6}$ Many reports have shown that natural products and their derivatives can protect against ROS-induced damage. Accordingly, they may have potential applications in preventing and/or curing diseases. ${ }^{7,8)}$ In recent years, traditional Chinese herbs have been found to possess excellent antioxidant activities ${ }^{9-14)}$; the phenolic and flavonoid components, in particular, have been identified as excellent antioxidants that have proven to be effective in scavenging free radicals. ${ }^{15-17)}$ Therefore, the use of herbs to inhibit oxidative stress and decrease damage from free radicals has garnered much attention.

More than 2000 herbs have been recorded in the Compendium of Materia Medica, one of the most famous ancient Chinese herb books, and most of these traditional Chinese herbs were applied in the form of multi-herb formulas in medical treatments and as dietary supplements. However, the application of herbal prescriptions today is mainly based on a doctor's experience and not on pharmacological mechanisms. ${ }^{18)}$ Traditional Chinese herb pairs (TCHPs), which are the basic unit in traditional Chinese prescriptions and an intermediate point between single herbs and multi-herb recipes, ${ }^{19)}$ consist of two relatively standard single herbs. Many herb pairs were recorded in the Treatise on Febrile and Miscellaneous Diseases and in the Synopsis of the Golden Chamber. According to Chinese records and the classic books about herbs in China, many TCHPs showed signifi- cantly better pharmacological efficacy than when the herbs were used individually. ${ }^{20-23)}$ For instance, the complete Qizhu Tang formula (composed of four herbs) was more effective in preventing cerebral oxidative injury in rats than any of the incomplete formulas or single herbs. ${ }^{24)} \mathrm{A}$ TCHP, $A s-$ tragalus membranaceus and Paeonia lactiflora (AME-PL), inhibited liver fibrosis in rats treated with carbon tetrachloride, and exhibited an increased hepato-protective capability relative to either of the herbs used individually. ${ }^{20)}$ However, the mechanisms by which the formulae and TCHPs achieve this synergism have not been elucidated, and changes to the combinations have rarely been studied. In order to investigate the mechanism of this synergy, six herbs were tested, including Radix Astragali (Astragalus membranaceus (AME)), Radix Paeoniae Alba (Paeonia lactiflora (PL)), Rhizoma Atractylodis Macrocephalae (Atractylodes macrocephala (AMA)), Radix Angelicae Sinensis (Angelica sinensis (AS)), Radix Glycyrrhizae (Glycyrrhiza uralensis (GU)) and Radix et Rhizoma Rhei (Rheum officinale (RO)), as well as eight TCHPs (AME-AS, AME-AMA, AME-RO, AME-GU, AMEPL, PL-AS, PL-AMA and PL-GU). The antioxidant activities of the TCHPs were compared with the theoretical sums of those of the respective constituent herbs as a means to evaluate the herb synergism. In addition, the influence of two different methods of combining the herbs in a TCHP, EM, the combination of extracts from the individual herbs, and $\mathrm{PM}$, the combination extract of a powdered mixture of the herbs, on the antioxidant ability of the herb pairs were compared for the first time. The correlation between the rate of enhancement in antioxidant capacity (REAC) and the rate of increase in phenolic and flavonoid contents (RIPC and RIFC) was studied as the preliminary means to quantify the synergistic effect. 


\section{MATERIALS AND METHODS}

Herbal Plants The six herbs, Radix Paeoniae Alba (Paeonia lactiflora (PL)), Rhizoma Atractylodis Macrocephalae (Atractylodes macrocephala (AMA)), Radix Angelicae Sinensis (Angelica sinensis (AS)), Radix Astragali (Astragalus membranaceus (AME)), Radix Glycyrrhizae (Glycyrrhiza uralensis (GU)) and Radix et Rhizoma Rhei (Rheum officinale (RO)), were obtained from Shijiazhuang Pharmaceutical Group of China and stored at room temperature.

Chemicals and Equipment 1,1-Diphenyl-2-picryl hydrazine (DPPH), Folin-Ciocalteau reagent, gallic acid, and rutin $(95 \%)$ were purchased from Sigma-Aldrich Chemical Co. (St. Louis, MO, U.S.A.). The hydroxyl radical and superoxide radical anion detection kits were purchased from the Nanjing Jiancheng Bioengineering Institute. All other chemical reagents were of analytical grade and obtained from Shanghai Chemical Reagent Co. Ultraviolet-visible (UVvis) spectroscopy was performed on a Scinco S-3100 spectrophotometer (Scinco Co., South Korea).

Preparation of Samples. Single Herb Samples All samples were powdered separately and sieved through a No. 40 mesh. The powder samples $(20 \mathrm{~g})$ were Soxhlet extracted with $150 \mathrm{ml}$ of ethanol for $2 \mathrm{~h}$. The extraction was repeated 3 times. The extracts were combined, evaporated under reduced pressure at $40^{\circ} \mathrm{C}$ and brought to a final volume of $100 \mathrm{ml}$ with ethanol. The solution was then centrifuged at $8000 \mathrm{rpm}$ for $15 \mathrm{~min}$, and the final samples were stored at $4{ }^{\circ} \mathrm{C}$ until they were analyzed.

Herb-Pair Samples In order to research the synergistic effects of the herb pairs, a powdered mixture containing equal proportions of two herbs ( $10 \mathrm{~g}$ each) was extracted according to the above method, yielding the powder mixture (PM) samples. Meanwhile, the extracts of two herbs were mixed in equal proportions, yielding the extract mixture (EM) samples.

DPPH Radical-Scavenging Assay The scavenging experiments with DPPH - were carried out as previously described by Rathee et al., ${ }^{25}$ with slight modifications. Briefly, $0.1 \mathrm{ml}$ samples of various herb concentrations in ethanol were added directly to $3.9 \mathrm{ml}$ of a DPPH - solution in ethanol $(0.1 \mathrm{~mm})$. The mixture was immediately shaken vigorously for $10 \mathrm{~s}$ on a vortex mixer, and then kept at $37^{\circ} \mathrm{C}$ for $30 \mathrm{~min}$. The absorbance was measured at $517 \mathrm{~nm}$, and the antioxidant capability (AA) was expressed as the percentage of DPPH reduced, which was calculated with the following formula:

$$
\mathrm{AA}_{\mathrm{DPPH}} \%=\left(\left(A_{\mathrm{B}}-A_{\mathrm{S}}\right) / A_{\mathrm{B}}\right) \times 100
$$

where $A_{\mathrm{S}}$ is the absorbance of the DPPH solution after reacting with the sample at a given concentration and $A_{\mathrm{B}}$ is the absorbance of the DPPH solution with an ethanol blank instead of a sample. The percentage of DPPH reduced was plotted against the concentration of each sample, and an $\mathrm{SC}_{50}$ value, which is defined as the concentration of the sample needed to scavenge $50 \%$ of the DPPH, was calculated from the graph.

Superoxide Anion Radical $\left(\mathrm{O}_{2}^{-\cdot}\right)$ Scavenging Assay We used the xanthine-xanthine oxidase $(\mathrm{X}-\mathrm{XO})$ enzymatic reaction to generate the superoxide radical anions, and the nitroblue tetrazolium (NBT) reduction assay was used to measure the superoxide anion radical scavenging capacity as previously described. ${ }^{26)}$ The tests were performed with a scav- enging $\mathrm{O}_{2}^{-} \cdot$ chemical kit (Nanjing Jiancheng Bioengineering Institute) according to the manufacturer's instructions. Briefly, the $\mathrm{O}_{2}^{-\cdot}$ generated by the xanthine/xanthine oxidase system reacts with NBT to form a colored formazan compound, which is proportional to the concentration of $\mathrm{O}_{2}^{-}$. Samples $(0.1 \mathrm{ml})$ of various herb concentrations were added to the reaction mixtures, and the reactions were kept at $37^{\circ} \mathrm{C}$ for $40 \mathrm{~min}$ and monitored spectrophotometrically at $550 \mathrm{~nm}$. Distilled water was used as a blank control. The measurement of each sample was repeated three times. The ability to scavenge $\mathrm{O}_{2}^{-} \cdot$ was expressed by the following formula:

$$
\mathrm{AA}_{\mathrm{O}_{2}^{-}} \%=\left(\left(A_{\mathrm{B}}-A_{\mathrm{S}}\right) / A_{\mathrm{B}}\right) \times 100
$$

where $A_{\mathrm{S}}$ is the absorbance of the reaction in the presence of an herbal sample, and $A_{\mathrm{B}}$ is the absorbance of the reaction mixture with ethanol as a blank control.

Hydroxyl Radical (·OH) Scavenging Assay Among all oxygen-centered radicals, the hydroxyl radical $(\cdot \mathrm{OH})$ is the most reactive and can damage numerous biomolecules. The hydroxyl radical $(\cdot \mathrm{OH})$ scavenging assay was performed by the method of Liu et al. ${ }^{27)}$ and measured with a commercially available detection kit (Nanjing Jiancheng Bioengineering Institute) according to the manufacturer's instructions. The hydroxyl radicals were generated by the Fenton reaction and then treated with a chromogenic substrate (NBT) to yield a stable colored substance, which was spectrophotometrically detected at $550 \mathrm{~nm}$. Each sample was investigated at various concentrations, and the $\mathrm{SC}_{50}$ value, which is the concentration of sample needed to scavenge $50 \%$ of the hydroxyl radicals, was calculated from the graph.

Determination of the Total Phenolic and Flavonoid Contents The determination of the total phenolic content was performed by the Folin-Ciocalteu method. ${ }^{28)}$ Briefly, $100 \mu \mathrm{l}$ of sample was mixed with $1 \mathrm{ml}$ of the Folin-Ciocalteu reagent (diluted ten-fold) and incubated at room temperature for $5 \mathrm{~min}$, and then $1 \mathrm{ml}$ of a $10 \% \mathrm{Na}_{2} \mathrm{CO}_{3}$ solution was added to the mixture. The absorbance was measured at $765 \mathrm{~nm}$ after a $90 \mathrm{~min}$ incubation at room temperature, and the results were expressed as gallic acid equivalents (GAE) per $\mathrm{ml}$ of extract.

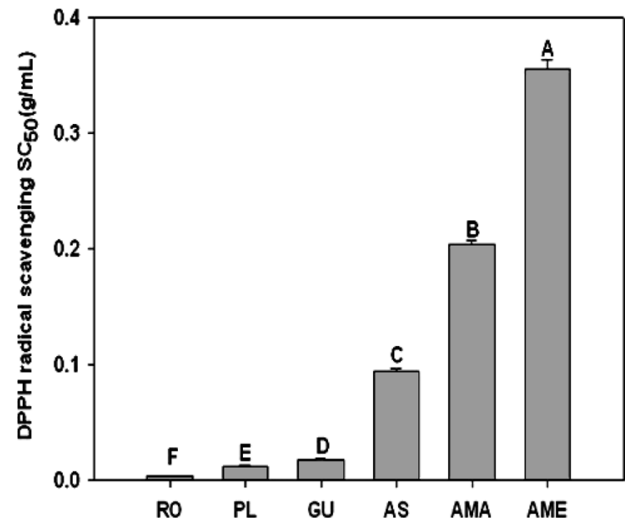

Fig. 1. The Measurement of DPPH Radical Scavenging Abilities of Six Herbs

The $\mathrm{SC}_{50}$ value is defined as the sample concentration needed to scavenge $50 \%$ of the DPPH. The herbs studied were RO (Rheum officinale), PL (Paeonia lactiflora), GU (Glycyrrhiza uralensis), AS (Angelica sinensis), AMA (Atractylodes macrocephala), and AME (Astragalus membranaceus). Data are expressed as the mean+standard deviation $(n=3)$. Mean values denoted with the same letter are not significantly different at the $5 \%$ level (Duncan's multiple range test). 
The total flavonoid content of the samples was determined according to the method described by $\mathrm{Li}$ et al. with some modifications. ${ }^{26)}$ The sample $(0.1 \mathrm{ml})$ was mixed with $0.3 \mathrm{ml}$ of a $5 \% \mathrm{NaNO}_{2}$ solution in a test tube and incubated for $5 \mathrm{~min}$, after which time $0.3 \mathrm{ml}$ of a $10 \% \mathrm{AlCl}_{3}$ solution was added and incubated for another $6 \mathrm{~min}$. The reaction was terminated by adding $2 \mathrm{ml}$ of a $1 \mathrm{M} \mathrm{NaOH}$ solution, and the absorbance of the mixture was measured immediately at $510 \mathrm{~nm}$. The total flavonoid content was expressed as the $\mathrm{mg}$ rutin equivalents (RE) per ml of extract.

Statistical Analysis All experiments were carried out in triplicate and the data are expressed as the mean \pm standard deviation. Statistical analysis was performed with the oneway analysis of variance (ANOVA) and the Duncan test. Correlation was analyzed with Bivariate Correlations (Spear-
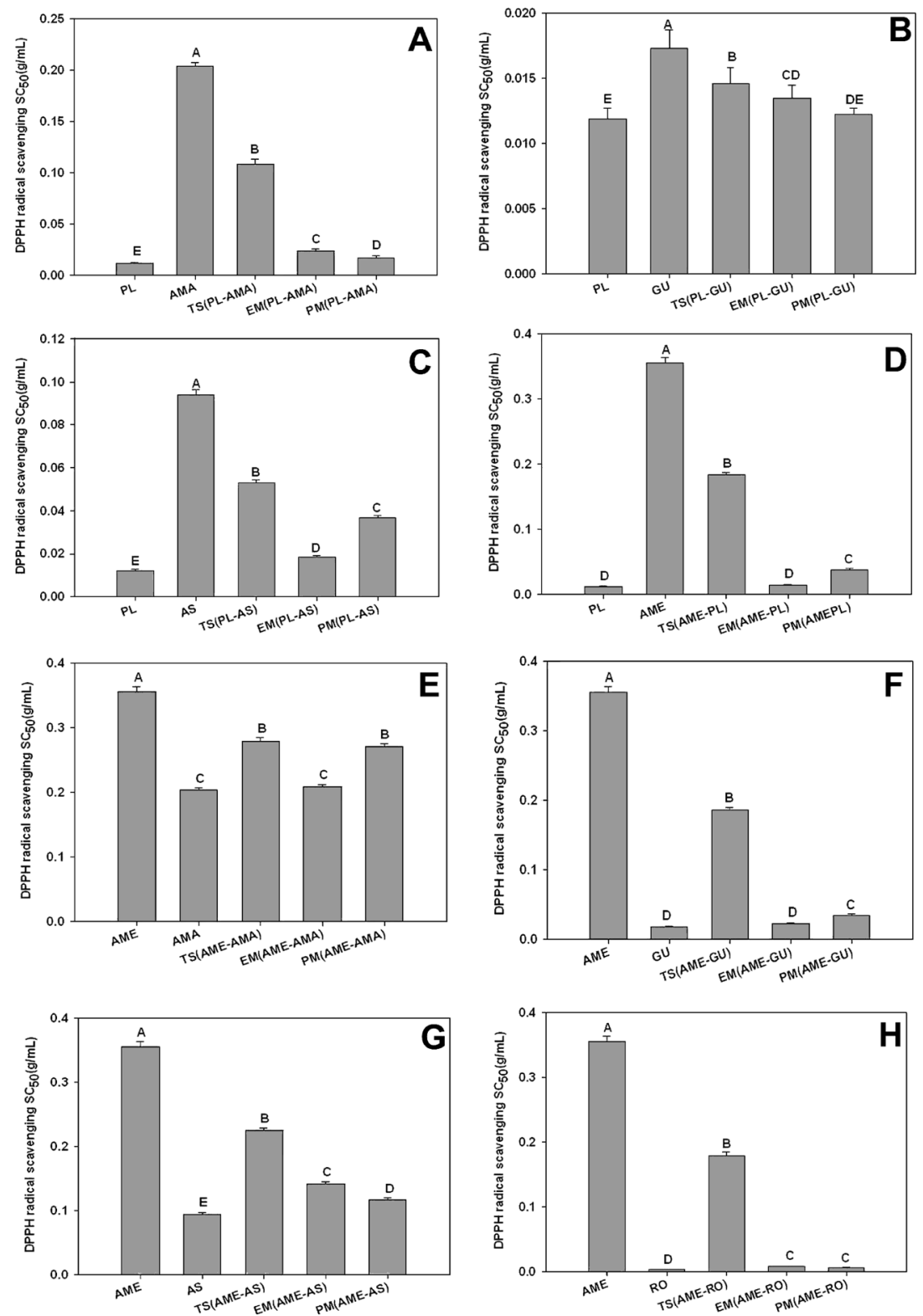

Fig. 2. The Measurement of DPPH Radical Scavenging Ability for Eight Traditional Chinese Herb Pairs (TCHPs)

The $\mathrm{SC}_{50}$ value is defined as the sample concentration needed to scavenge $50 \%$ of the DPPH. The theoretical sum of the DPPH radical scavenging abilities of the respective herbs comprising each pair is denoted as TS. The samples from the two methods of combining the herbs are described as EM (the combination of extracts from the individual herbs) and PM (the extract of a powdered mixture of the herbs). The herbs used in the TCHPs are RO (Rheum officinale), PL (Paeonia lactiflora), GU (Glycyrrhiza uralensis), AS (Angelica sinensis), AMA (Atractylodes macrocephala) and AME (Astragalus membranaceus). The pairs are (A) PL-AMA, (B) PL-GU, (C) PL-AS, (D) AME-PL, (E) AMEAMA, (F) AME-GU, (G) AME-AS and (H) AME-RO. The data are expressed as the mean \pm standard deviation $(n=3)$. Mean values denoted with the same letter are not significantly different at the $5 \%$ level (Duncan's multiple range test). 
the abilities of the six individual herbs to scavenge DPPH radicals were evaluated. The results revealed that the DPPH scavenging activity of all the samples was concentration dependent. As shown in Fig. 1, RO had the highest DPPH radical scavenging efficiency $\left(\mathrm{SC}_{50}=3.95 \mathrm{mg} / \mathrm{ml}\right)$, while $\mathrm{AME}$ and AMA had the weakest scavenging ability $\left(\mathrm{SC}_{50}=\right.$ $355.34 \mathrm{mg} / \mathrm{ml}$ and $\mathrm{SC}_{50}=203.95 \mathrm{mg} / \mathrm{ml}$, respectively). The other three herbs had activities in the order $\mathrm{PL}>\mathrm{GU}>\mathrm{AS}$ $\left(\mathrm{SC}_{50}=11.91 \mathrm{mg} / \mathrm{ml}, \mathrm{SC}_{50}=17.33 \mathrm{mg} / \mathrm{ml}\right.$, and $\mathrm{SC}_{50}=93.92$ $\mathrm{mg} / \mathrm{ml}$, respectively). These results showed the herbs used in the eight TCHPs had a wide range of antioxidant activities.

DPPH Radical Scavenging Capabilities of the Eight TCHPs Previous studies have shown that the potency of a combination of herbs in a formula does not result from a quantitative addition of the potencies of the individual herbs, but rather from a more complex interaction among herbs with different pharmacological functions. ${ }^{22}$ For example, $\mathrm{Yan}^{34)}$ reported that the chemical ingredients were greatly altered when three medicinal herbs were decocted together, and that during the preparation of a decoction, a new compound, 5-hydroxymethyl-2-furaldehyde (5-HMF), was produced. It was later found to have a strong antioxidant effect in vivo. Such studies indicated that the process by which the herbs are combined might contribute to their antioxidant capability. In order to probe the synergistic effect of the antioxidant activities of the herb pairs, we selected eight TCHPs with two combination methods each (PM and EM) to investigate the influence of the combination method on antioxidant ability. The results show that all eight TCHPs have antioxidant activities greater in the DPPH radical scavenging assay than the theoretical sum of those of the respective constituent herbs $(p<0.05)$ (Fig. 2), indicating a synergistic effect in these herb pairs. Most interestingly, we found that the AMEGU, AME-PL and AME-AMA combinations demonstrated antioxidant activities that were not significantly different from those of the herb in the pair that had the higher antioxidant activity $(p>0.05)$ (EM (AME-PL): $\mathrm{SC}_{50}=0.0145$ and

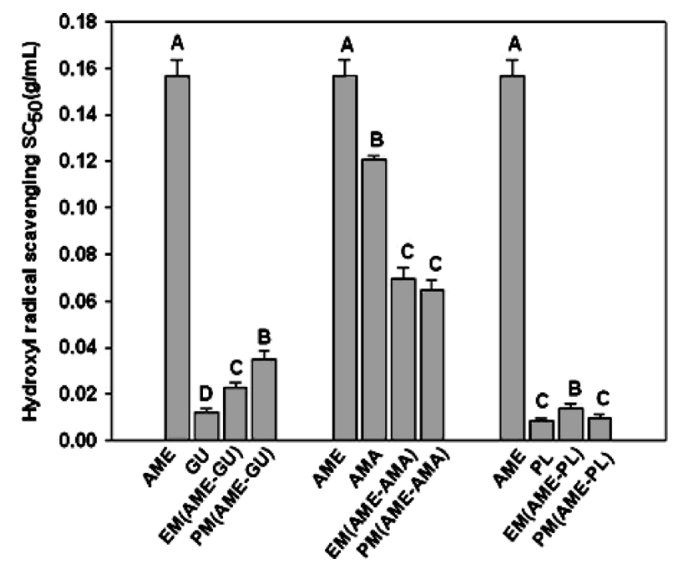

Fig. 3. The Measurement of Hydroxyl Radical $(\cdot \mathrm{OH})$ Scavenging Ability for Three Traditional Chinese Herb Pairs (TCHPs)

The $\mathrm{SC}_{50}$ value is defined as the sample concentration needed to scavenge $50 \%$ of the hydroxyl radicals. The samples from the two methods of combining the herbs are described as EM (the combination of extracts from the individual herbs) and PM (the extract of a powdered mixture of the herbs). The herbs used in the TCHPs were AME (Astragalus membranaceus), GU (Glycyrrhiza uralensis), AMA (Atractylodes macrocephala), and PL (Paeonia lactiflora). The pairs examined were AME-GU, AME-PL and AME-AMA. Data are expressed as the mean \pm standard deviation $(n=3)$. Mean values denoted with the same letter are not significantly different at the 5\% level (Duncan's multiple range test).
PL: $\mathrm{SC}_{50}=0.0119$; EM (AME-GU): $\mathrm{SC}_{50}=0.0222$ and $\mathrm{GU}$ : $\mathrm{SC}_{50}=0.0173$; $\mathrm{EM}$ (AME-AMA): $\mathrm{SC}_{50}=0.2085$ and AMA: $\mathrm{SC}_{50}=0.2039$ ). Furthermore, the two combination methods, $\mathrm{PM}$ and $\mathrm{EM}$, do influence the antioxidant activities of the herb pairs $(p<0.05)$. The $\mathrm{SC}_{50} \mathrm{~s}$ for the $\mathrm{PM}$ samples were as follows: PM (PL-AMA)>EM (PL-AMA), PM (AMEAS) $>$ EM (AME-AS), while those for the EM samples were as follows: EM(PL-AS) $>$ PM (PL-AS), EM (AME-PL) $>$ PM (AME-PL), EM (AME-AMA) $>$ PM (AME-AMA) and EM (AME-GU) $>$ PM (AME-GU) (Fig. 2). These results also suggest that an appropriate method of combining the herbs should be considered in herb pair therapy in order to attain the best antioxidant activity.

Hydroxyl Radical Scavenging Ability of AME-PL, AME-GU and AME-AMA Hydroxyl radicals are extremely reactive, short-lived species that can hydroxylate DNA, proteins and other biomolecules. ${ }^{35)}$ Thus, it is important to assess the hydroxyl radical scavenging ability of the herbal samples. In the present study, the synergistic effect of the AME-PL, AME-GU and AME-AMA combinations in
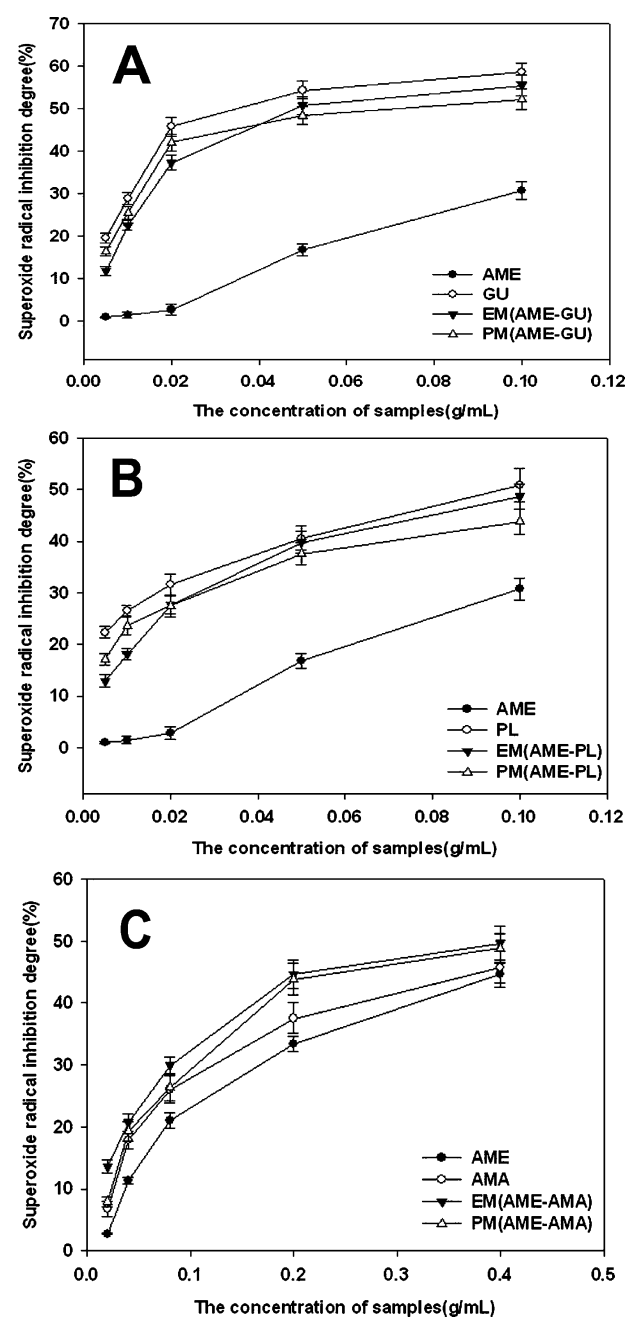

Fig. 4. The Measurement of Concentration-Dependent Superoxide Radical Anion $\left(\mathrm{O}_{2}^{-} \cdot\right)$ Scavenging Abilities of Three Traditional Chinese Herb Pairs (TCHPs)

The samples from the two methods of combining the herbs are described as EM (the combination of extracts from the individual herbs) and PM (the extract of a powder mixture of the herbs). The herbs used in the TCHPs were AME (Astragalus membranaceus), AMA (Atractylodes macrocephala), GU (Glycyrrhiza uralensis) and PL (Paeonia lactiflora). The pairs examined were (A) AME-GU, (B) AME-PL and (C) AME-AMA. Data are expressed as the mean \pm standard deviation $(n=3)$. 
scavenging DPPH radicals was quite significant $(p<0.05)$, while their $\mathrm{SC}_{50} \mathrm{~S}$ were not significantly different when compared to those of the individual herb in the pair that had the higher antioxidant ability (Figs. 2A-H). The three herb pairs mentioned above were selected for evaluation in terms of their ability to scavenge hydroxyl radicals. The results show that the three herb pairs also demonstrate a synergistic effect in hydroxyl radical scavenging. Interestingly, the AME-AMA combination prepared by both the EM and PM methods demonstrated a significantly higher hydroxyl radical scavenging activity than the herbs used individually $(p<$ 0.05) (Fig. 3).

Superoxide Radical Anion Scavenging Capability of AME-PL, AME-GU and AME-AMA The superoxide radical anion $\left(\mathrm{O}_{2}^{-\cdot}\right)$ has been the subject of intense interest lately due to our increased understanding of its in vivo importance in different diseases. Although $\mathrm{O}_{2}^{-} \cdot$ is far less active than $\cdot \mathrm{OH}$, it can generate $\cdot \mathrm{OH}$ by means of superoxide dismutase (SOD) in biological systems. Thus, a superoxide radical anion $\left(\mathrm{O}_{2}^{-} \cdot\right)$ scavenging assay was also used to evaluate the antioxidant ability of these three herb pairs. The extracts were tested at different concentrations and the results showed that the scavenging abilities of all the extracts were concentration dependent (Figs. 4A-C). As shown in Fig. 4A, AME has the lowest $\mathrm{O}_{2}^{-} \cdot$ scavenging ability, while GU has the highest scavenging ability, which is just slightly higher than that of the EM (AME-GU) and PM (AME-GU) samples. There was no significant difference among GU, EM (AME$\mathrm{GU})$ and PM (AME-GU) at a concentration of $0.05 \mathrm{~g} / \mathrm{ml}$. The activity of the AME-PL combination was similar to that of the AME-GU combination (Fig. 4B). As with the hydroxyl radical scavenging assay, both the EM and $\mathrm{PM}$ preparations of the AME-AMA combination $(0.2 \mathrm{~g} / \mathrm{ml})$ had significantly higher superoxide radical anion scavenging ability than the herbs used individually (Fig. 4C).

Determination of Total Phenolic and Flavonoid Con- tents in the Eight TCHPs As is well known, the phenolic and flavonoid compounds in natural substances, such as herbs, fruits and vegetables, have excellent antioxidant qualities. The content of total phenolic and flavonoid has been shown to be positively correlated with antioxidant ability. ${ }^{16,36)}$ Therefore, the variation in the contents of phenolic and flavonoid compounds was quantitatively measured to elucidate the potential mechanism of this synergistic effect. As shown in Table 1, the total phenolic and flavonoid contents of the eight TCHPs exhibited various differences from their respective theoretical sums, which may be a reason for the synergistic effect of the herb's antioxidant activities. The correlation between the rate of enhancement in antioxidant capacity (REAC) and the rate of increase in phenolic and flavonoid contents (RIPC and RIFC) in these eight TCHPs was further investigated. REAC was calculated as the rate of enhancement in the $\mathrm{SC}_{50}$ of the herb pairs as compared to the respective theoretical sum of their $\mathrm{SC}_{50} \mathrm{~S}$ in the DPPH radical scavenging assay, while the RIPC and RIFC were calculated as the rate of increase in phenolic and flavonoid contents in the herb pairs as compared to their respective theoretical sum. These results showed that the significant correlation between REAC and RIFC was higher than the correlation between REAC and RIPC (the Spearman correlation coefficients were: $R_{\text {REAC-RIFC }}=0.559, p=0.024$; and $R_{\text {REAC-RIPC }}=0.382, p=0.144$, respectively), which implied that the changes in total flavonoid content might make a more significant contribution to the synergism.

A previous study on the synergism displayed by ascorbic acid and $\alpha$-tocopherol interpreted this synergism as resulting from the regeneration of $\alpha$-tocopherol from $\alpha$-chromanoxyl radicals that are formed by the interaction of $\alpha$-tocopherol with DPPH, with the aid of ascorbic acid. ${ }^{37)}$ Other similar synergisms have also been discovered, such as the synergisms between EGCG and $\alpha$-tocopherol, rosmarinic acid and $\alpha$-tocopherol, and procyanidins and $\alpha$-tocopherol. ${ }^{38-40)}$ An-

Table 1. The Rate of Increase in Phenolic, Flavonoid Contents and the Rate of Enhancement in DPPH Scavenging Ability in the Herbs and the TCHPs

\begin{tabular}{|c|c|c|c|c|c|}
\hline & Phenolics content $(\mathrm{mg} \cdot \mathrm{GAE} / \mathrm{ml})$ & $\operatorname{RIPC}^{a)}(\%)$ & Flavonoids content $(\mathrm{mg} \cdot \mathrm{RE} / \mathrm{ml})$ & $\operatorname{RIFC}^{b)}(\%)$ & $\operatorname{REAC}^{c)}(\%)$ \\
\hline AS & $0.327 \pm 0.031$ & - & $0.215 \pm 0.022$ & - & - \\
\hline AME & $0.265 \pm 0.022$ & - & $0.094 \pm 0.008$ & - & - \\
\hline AMA & $0.160 \pm 0.023$ & - & $0.115 \pm 0.011$ & - & - \\
\hline PL & $0.795 \pm 0.055$ & - & $0.100 \pm 0.011$ & - & - \\
\hline GU & $1.340 \pm 0.132$ & - & $0.504 \pm 0.044$ & - & - \\
\hline RO & $2.898 \pm 0.313$ & - & $1.976 \pm 0.093$ & - & - \\
\hline PM (AME-GU) & $0.822 \pm 0.063$ & 2.421 & $0.308 \pm 0.015$ & 2.972 & 81.742 \\
\hline EM (AME-GU) & $0.940 \pm 0.068$ & 17.119 & $0.316 \pm 0.079$ & 5.808 & 88.064 \\
\hline PM (AME-AMA) & $0.070 \pm 0.002$ & -66.998 & $0.106 \pm 0.012$ & 1.149 & 3.210 \\
\hline EM (AME-AMA) & $0.234 \pm 0.021$ & 10.116 & $0.107 \pm 0.008$ & 2.034 & 25.451 \\
\hline PM (AME-AS) & $0.245 \pm 0.016$ & -17.290 & $0.129 \pm 0.013$ & -16.585 & 48.167 \\
\hline EM (AME-AS) & $0.324 \pm 0.027$ & 9.533 & $0.149 \pm 0.009$ & -3.444 & 37.224 \\
\hline PM (AME-RO) & $1.593 \pm 0.133$ & 0.741 & $1.015 \pm 0.075$ & -1.918 & 96.251 \\
\hline EM (AME-RO) & $1.981 \pm 0.165$ & 25.277 & $1.179 \pm 0.081$ & 13.932 & 95.547 \\
\hline PM (AME-PL) & $0.547 \pm 0.037$ & 3.168 & $0.103 \pm 0.009$ & 5.745 & 79.175 \\
\hline EM (AME-PL) & $0.562 \pm 0.041$ & 5.937 & $0.149 \pm 0.016$ & 52.636 & 92.109 \\
\hline PM (PL-GU) & $0.724 \pm 0.052$ & -32.199 & $0.196 \pm 0.018$ & -35.254 & 16.348 \\
\hline EM (PL-GU) & $1.134 \pm 0.096$ & 6.174 & $0.309 \pm 0.032$ & 2.042 & 7.934 \\
\hline PM (PL-AMA) & $0.448 \pm 0.035$ & -6.322 & $0.155 \pm 0.01$ & 43.801 & 84.379 \\
\hline EM (PL-AMA) & $0.470 \pm 0.031$ & -1.587 & $0.162 \pm 0.009$ & 50.076 & 78.328 \\
\hline PM (PL-AS) & $0.439 \pm 0.036$ & -21.778 & $0.134 \pm 0.012$ & -14.914 & 30.773 \\
\hline EM (PL-AS) & $0.574 \pm 0.041$ & 2.343 & $0.180 \pm 0.021$ & 13.921 & 65.226 \\
\hline
\end{tabular}

Data are in mean \pm S.D., $n=3$ ). a) The rate of increase in the phenolic content. $b$ ) The rate of increase in the flavonoid content. $c$ ) The rate of enhancement in antioxidant ability. - Not calculated. 
other possible source of the synergistic effects on the radical scavenging activities are plant polyphenols containing a 3,4dihydroxy substructure. ${ }^{41)}$ Chaillou and Nazareno demonstrated that the antioxidant capabilities of polyphenols were not only related to the number of phenolic hydroxyls but also to their position. ${ }^{42)}$ With respect to this idea, the synergistic effects of the eight TCHPs might be correlated not only with RIFC, but also with other new active components or with changes in the structure of some polyphenols that may be altered to contain a 3,4-dihydroxy substructure. This hypothesis needs further study by means of infrared spectroscopy, liquid chromatography-mass spectrometry, and nuclear magnetic resonance analysis techniques.

Acknowledgements We are grateful to Prof. Ling-chuan $\mathrm{Xu}$ (Shandong University of Traditional Chinese Medicine) for his generous support.

\section{REFERENCES}

1) Mitsuyama S., Nippon Rinsho, 65 (Suppl. 4), 243-250 (2007).

2) Castelao J. E., Gago-Dominguez M., Med. Hypotheses, 71, 39-44 (2008).

3) Czaja M. J., Semin. Liver Dis., 27, 378-389 (2007).

4) Gupta S., Sarotra P., Aggarwal R., Dutta N., Agnihotri N., Dig. Dis. Sci., 52, 3092-3098 (2007).

5) Ishii N., Cornea, 26, S3-S9 (2007).

6) Ranjbar A., Khorami S., Safarabadi M., Shahmoradi A., Malekirad A. A., Vakilian K., Mandegary A., Abdollahi M., Evid.-Based Compl. Alt. Med., 3, 469-473 (2006).

7) Cuzzocrea S., Riley D. P., Caputi A. P., Salvemini D., Pharmacol. Rev., 53, 135-159 (2001).

8) Capecka E., Mareczek A., Leja M., Food Chem., 93, 223-226 (2005).

9) Xi M. M., Hai C. X., Tang H. F., Chen M. S., Fang K. Q., Liang X., Phytother. Res., 22, 228-237 (2008).

10) Yoo K. M., Lee C. H., Lee H., Moon B., Lee C. Y., Food Chem., 106, 929-936 (2008).

11) Cai Y. Z., Luo Q., Sun M., Corke H., Life Sci., 74, 2157-2184 (2004).

12) Yen F., Wu T., Lin L., Cham T., Lin C., Food Chem., 108, 455-462 (2008).

13) Han J., Weng X. C., Bi K. S., Food Chem., 106, 2-10 (2008).

14) Pan Y. M., Liang Y., Wang H. S., Liang M., Food Chem., 88, 347350 (2004).

15) Chen H.-Y., Lin Y.-C., Hsieh C.-L., Food Chem., 104, 1418-1424 (2007).

16) Kim H. J., Chang E. J., Cho S. H., Chung S. K., Park H. D., Choi S W., Biosci. Biotechnol. Biochem., 66, 1990-1993 (2002).
17) Ao C., Li A., Elzaawely A. A., Xuan T. D., Tawata S., Food Control, 19, 940-948 (2008).

18) Ung C. Y., Li H., Cao Z. W., Li Y. X., Chen Y. Z., J. Ethnopharmacol., 111, 371-377 (2007).

19) Li X. R., Liang Y. Z., Guo F. Q., Acta Pharmacol. Sin., 27, 491-498 (2006).

20) Sun W. Y., Wei W., Wu L., Gui S. Y., Wang H., J. Ethnopharmacol., 112, 514-523 (2007).

21) Xue L., Zhang H. Y., Qin L., Wang X. C., Wang L., China J. Chinese Mat. Med., 25, 175-178 (2000).

22) Jia W., Gao W. Y., Yan Y. Q., Wang J., Xu Z. H., Zheng W. J., Xiao P. G., Phytother. Res., 18, 681-686 (2004).

23) Xue J. X., Yan Y. Q., Jiang Y., China J. Chinese Mat. Med., 19, 108 110, 128 (1994).

24) Wang X. J., Ichikawa H., Konishi T., Biol. Pharm. Bull., 24, 558-563 (2001).

25) Rathee J. S., Patro B. S., Mula S., Gamre S., Chattopadhyay S., J. Agric. Food Chem., 54, 9046-9054 (2006).

26) Li Y. F., Guo C. J., Yang J. J., Wei J. Y., Xu J., Cheng S., Food Chem., 96, 254-260 (2006).

27) Liu C. S., Cheng Y., Hu J. F., Zhang W., Chen N. H., Zhang J. T., Acta Pharmacol. Sin., 27, 1137-1145 (2006).

28) Reynertson K. A., Yang H., Jiang B., Basile M. J., Kennelly E. J., Food Chem., 109, 883-890 (2008).

29) Wang X. J., Feng P., Acta Pharmacol. Sin., 21, 1141-1144 (2000).

30) Chan J. Y., Leung P. C., Che C. T., Fung K. P., Phytother. Res., 22, 190-196 (2008).

31) Wang D., Shen W., Tian Y., Dong Z., Liu G., Sun Z., Yang S., Zhou S., China J. Chinese Mat. Med., 20, 240-242, 254 (1995).

32) Lee S. C., Kwon Y. S., Son K. H., Kim H. P., Heo M. Y., Arch. Pharm. Res., 28, 775-783 (2005).

33) Wang D., Shen W., Tian Y., Sun Z., Jiang C., Yuan S., China J. Chinese Mat. Med., 21, 746-748, 763 (1996).

34) Yan Y. Q., World Science and Technology-Modern Trad. Chin. Med., 3, 17-20 (2001).

35) Du J., Gebicki J. M., Int. J. Biochem. Cell Biol., 36, 2334-2343 (2004).

36) Ayoola G. A., Folawewo A. D., Adesegun S. A., Abioro O. O., Adepoju-Bello A. A., Coker H. A. B., Afr. J. Plant Sci., 2, 124-128 (2008).

37) Niki E., Tsuchiya J., Tanimura R., Kamiya Y., Chem. Lett., 11, 789 792 (1982).

38) Maffei Facino. R., Carini M., Aldini G., Calloni M. T., Bombardelli E., Morazzoni P., Planta Med., 64, 343-347 (1998).

39) Hu C., Kitts D. D., Mol. Cell. Biochem., 218, 147-155 (2001).

40) Jayasinghe C., Gotoh N., Aoki T., Wada S., J. Agric. Food Chem., 51, 4442-4449 (2003)

41) Saito S., Kawabata J., J. Agric. Food Chem., 52, 8163-8168 (2004).

42) Chaillou L. L., Nazareno M. A., J. Agric. Food Chem., 54, 83978402 (2006). 